\title{
Effect of the Unsteadiness on the Diffuser Flow in a Transonic Centrifugal Compressor Stage
}

\author{
N. Bulot and I. Trébinjac \\ Laboratoire de Mécaniques des Fluides et d'Acoustique, Ecole Centrale de Lyon, ECL/UCBL/INSA/UMR CNRS 5509, \\ 69134 Ecully Cedex, France \\ Correspondence should be addressed to N. Bulot, nicolas.bulot@ec-lyon.fr
}

Received 4 September 2008; Revised 5 February 2009; Accepted 19 March 2009

Recommended by Chunill Hah

The study is focused on the analysis of the flow structure within the vaned diffuser of a transonic high-pressure centrifugal compressor stage. The analyzed time-dependent flow field comes from unsteady computations of the stage using a 3D NavierStokes code with a phase-lagged technique, at an operating point close to the design point. A good comparison with available experimental data allowed the use of CFD for investigating the details of the flow in order to assess the effect of the unsteadiness in the diffuser flow development. Applying various data processing techniques, it is shown that the unsteadiness is due to the jet and wake flow structure emerging from the radial impeller and to the pressure waves brought about by the interaction between the vane bow shock wave and the impeller blade. The interaction between the pressure waves and the vane pressure side boundary layer leads to a pulsating behavior of separated bubbles within the diffuser. The pressure waves are similar in shape and strength whatever the blade height. The observed change in the flow field from hub to tip is due to migration of the low momentum fluid contained in the wake toward the pressure side/hub corner.

Copyright ( 2009 N. Bulot and I. Trébinjac. This is an open access article distributed under the Creative Commons Attribution License, which permits unrestricted use, distribution, and reproduction in any medium, provided the original work is properly cited.

\section{Introduction}

Impeller-diffuser interaction in centrifugal compressors with vaned diffuser plays an important role in the compression process. The vaned diffuser has to tolerate the distorted upstream flow due to jet-wake structure coming from the impeller, whereas the impeller is submitted to the potential effect of the vaned diffuser.

Actually, the majority of the studies dedicated to impeller-diffuser interaction are focused on the overall performances of the diffuser. Krain [1], Inoue and Cumpsty [2], and Ziegler et al. [3, 4] tested different diffuser configurations to show the effect of different parameters on the pressure recovery through the diffuser. The main result is the importance of the inlet axisymetric flow angle which is governed by the radial gap between impeller blade trailing edge and diffuser vane leading edge. However, all the tested compressors had subsonic conditions at diffuser inlet.

Deniz et al. [5] realized experimental studies of two vaned diffusers with variable upstream flow conditions. They have confirmed that the diffuser performances are essentially correlated with the inlet flow angle (by means of linear relation), even at supersonic upstream conditions. Nevertheless, the generated upstream flow was axisymetric and therefore unable to model the impeller outlet flow distortion due to the jet-wake structure.

In the works which are above-mentioned, unsteadiness had no significant effect on the performances because the compressors were low loaded. However for industrial centrifugal compressors, especially in aeronautical domain, the technical constraints (low weight, small size, high performances, etc.) lead to increase the compressor loading and specific speed. Therefore, the blade passage frequency increases and the flow emerging from the impeller is even more distorted because of the loading increase. Consequently the unsteady flow structures may play an important role from an energetic point of view.

Shum et al. [6] provided a detailed numerical analysis of the unsteady potential effect (due to the vaned diffuser) on the impeller tip leakage flow, within a high loaded centrifugal compressor. They have shown that it exists an optimum size of the radial gap to provide an optimum pressure ratio. 


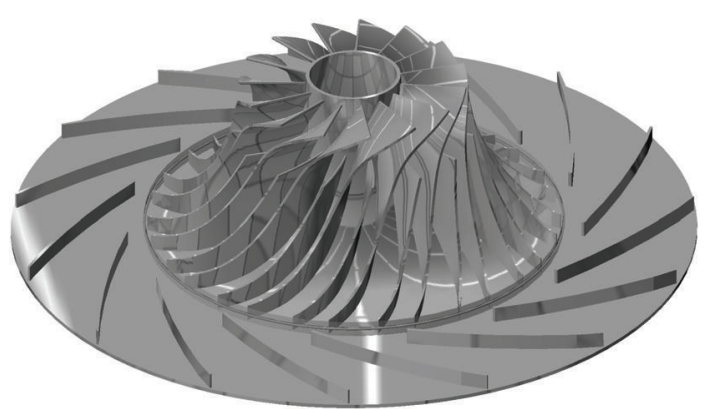

Figure 1: 3D sketch of the centrifugal compressor stage.

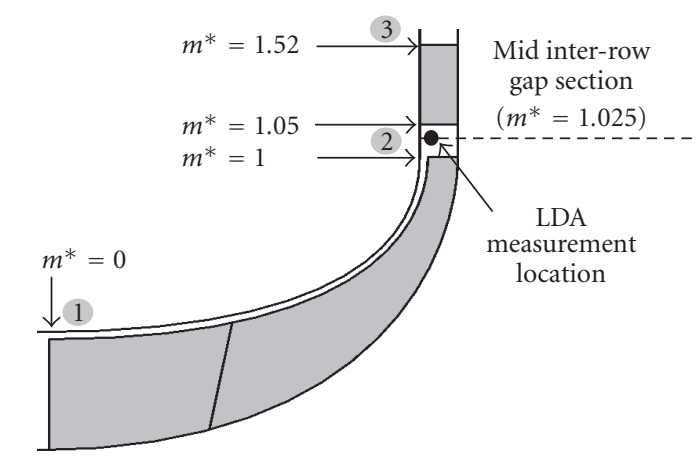

Figure 2: Meridional view of the compressor stage.

Krain [7] and Krain and Hah [8] investigated the unsteady flow field in high-pressure-ratio centrifugal compressor. Both the experimental and numerical results have shown a high level of unsteadiness in the interrow gap, and a quasisteady behavior of the flow through the vaned diffuser passage. Actually, the relatively large radial gap allowed an efficient mixing process which minimized the interaction phenomena. Moreover, the vaned diffuser was fully shocked and the shock did not go upstream the vaneless diffuser.

Beside the contribution of the impeller-diffuser interaction to the overall performance, the interaction may lead to instabilities all the more that the operating point approaches the surge line [9-11].

Due to the fact that the diffuser is thought to play an important role in the compression system and to impact the aerodynamic efficiency significantly, attention is focused on this sensible component. The aim of the present paper is to reach a comprehensive description of the underlying mechanisms involved in the interaction at a stable operating point and to give a detailed description of the unsteadiness inside the diffuser.

\section{Test Case}

The test case is a centrifugal compressor stage designed and built by Turbomeca. The backswept unshrouded impeller is composed of $N_{R}$ main blades and $N_{R}$ splitter blades. The vaned diffuser is composed of $N_{S}$ vanes, which leading edges

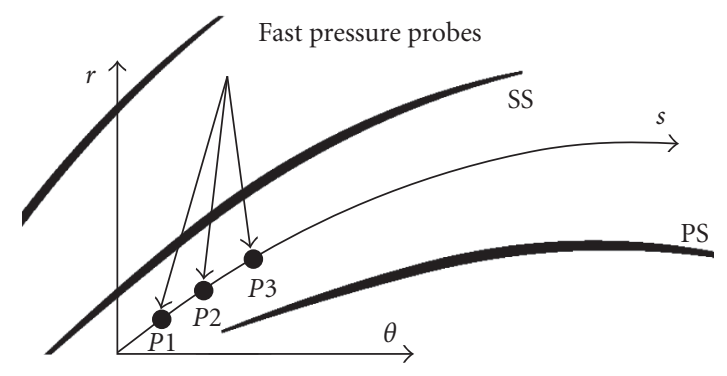

FIGURE 3: Location of fast pressure sensors in the diffuser passage.

are located at $105 \%$ of the radius of the impeller blade trailing edges. A 3D sketch and a meridional view of the compressor stage are given in Figures 1 and 2, respectively.

The health of the facility during the test was continuously monitored via the measured pressures, temperatures, and flow rate which are given at $\pm 0.4 \%, \pm 1 \mathrm{~K}$, and $\pm 0.2 \%$, respectively. LDA measurements were performed at crosssections from the impeller inlet up to the diffuser throat and could be found in [12]. Some LDA data acquired at mid inter row gap (Figure 2) are shown in this paper. Finally, the unsteady pressure was measured with 15 fast pressure sensors located in the shroud at mid inter row gap and within the diffuser vane passage. Some results obtained within the diffuser passage at three positions along a mid passage streamline (Figure 3 ) are given in this paper.

\section{Numerical Simulation}

The computations were performed with the elsA software developed at ONERA, which is more precisely described by Cambier and Gazaix [13]. The code solves the compressible RANS equations associated with the two equations $(k-l)$ turbulence model of Smith [14] on multiblock structured grids. The equations are discretized in the relative rotating frame using Jameson's centered space scheme in a "cellcentered" approach. In order to stabilize the scheme, 2ndorder and 4th-order dissipative terms are added at the 4 steps of the Runge-Kutta time-integration scheme. An implicit residual smoothing technique is added to this explicit scheme.

For the impeller-diffuser interaction computation, the phase-lagged approach is used. In this approach, the computation domain is limited to a single blade passage for each row. Assuming that there are no external unsteady causes, the unsteady effects are only due to the rotation. Then, the flow is time-periodic in the frame of reference of the rows, $T_{S}=2 \pi / \Omega_{R} N_{R}$ being the period in the diffuser frame and $T_{R}=2 \pi / \Omega_{R} N_{S}$ being the period in the impeller frame. As a consequence of the time-periodicity in each frame, a phaselag exists between two adjacent blade passages. For each row, this phase lag is the time taken by a blade of the next row to cover the pitch of the row, modulo the time-period of the row. Basically, the phase-lagged technique consists in storing the flow values on the periodic boundaries and on the 


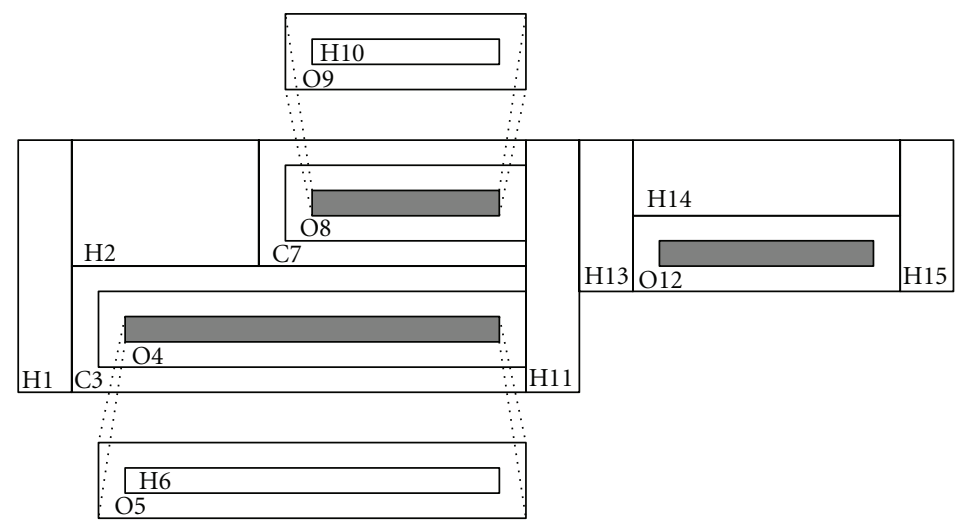

FIGURE 4: Stage mesh topology.

impeller-diffuser interface boundaries in order to deal with the phase lag existing between adjacent blade passages.

One unsteady computation needed at least 23 rotating wheel revolutions to get good convergence. It led to more than 100 hours CPU on NEC-SX8.

3.1. Stage Mesh. A multidomain approach on structured meshes used $\mathrm{H}, \mathrm{C}$, and $\mathrm{O}$ topologies that are employed (Figure 4). The near-wall region around the blades is described by an O-block with $y^{+}<3$, to allow a precise description of the viscous effects and to capture the turbulent gradients. C-blocks are used to connect O-blocks of the main blade and the splitter. Upstream, downstream, and interface regions are defined with classical $\mathrm{H}$-blocks. The tip clearance is meshed with an $\mathrm{O}-\mathrm{H}$ topology to provide a good connexion with the O-block around the blades.

The complete stage mesh is composed of 15 blocks including 1,093,851 nodes in the impeller blade passage and 618,192 nodes in the diffuser vane passage.

\section{Overall Results}

The pressure ratio $\left(\pi=p_{3} / p_{01}\right)$ curves coming from the unsteady calculations and the experiments are plotted in Figure 5 as a function of the specific mass flow $\left(\dot{m}_{s p}=\right.$ $\dot{m} \sqrt{\gamma R T_{01}} / D_{1 s}^{2} p_{01}$ ), at rotor speed $\Omega_{R}=0.927 . \Omega_{\text {nom }}$.

The experimental values of $p_{3}$ are the mean values of the static pressures measured on the shroud and hub wall surfaces at the stage outlet (Figure 2). The numerical values come from time and area-averaged values extracted in the same outlet section. An excellent agreement between the unsteady numerical data and the experiments is found for the five calculated points. It has to be noted that operating points neither close to the compressor stall nor close to choke were calculated in the present study.

In order to reach a comprehensive description of the underlying mechanisms involved in the interaction, attention is now focused on the time-dependent flow in the diffuser entry and diffuser passages, at a given operating point which is circled in Figure 5. This point was chosen because it is the peak efficiency operating point. Moreover, at this

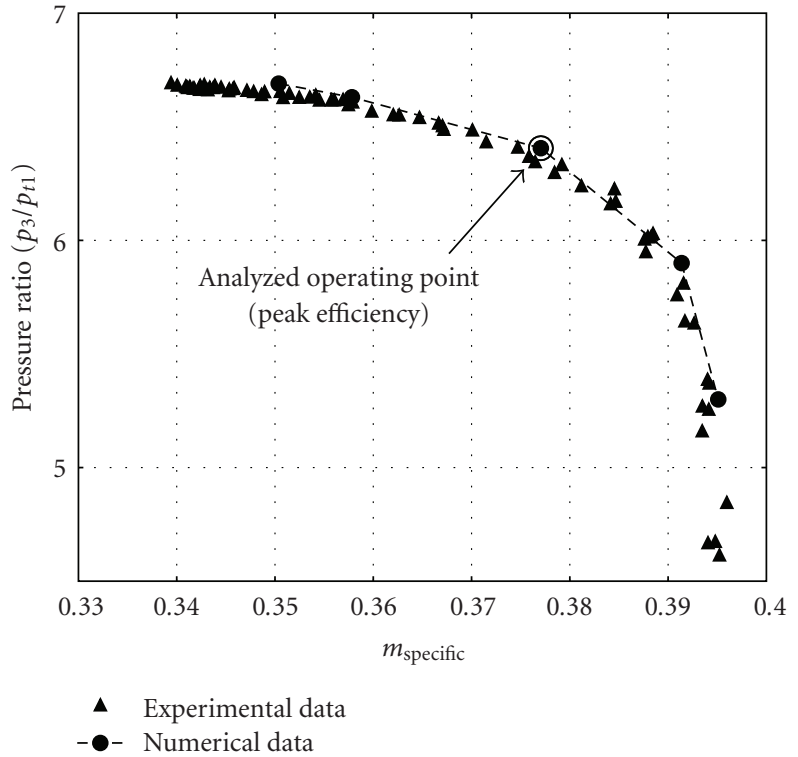

Figure 5: Pressure ratio of the compressor stage $\left(\Omega_{R}=\right.$ 0.927. $\left.\Omega_{\text {nom }}\right)$.

point, experimental internal flow data were available and the unsteady calculation exhibited an excellent convergence rate.

The good agreement between numerical and experimental results not only is achieved concerning the overall performances but is also confirmed regarding the internal flow pattern. A first example is given in Figure 6 which gives the reduced relative velocity $\left(W / U_{\text {ref }}\right)$ at mid inter row gap and $70 \%$ section height, over a stator pitch and a rotor time period. Figures 6(a) and 6(b) show the experimental and numerical results, respectively. The vertical white line represents a diffuser vane location whereas the two oblique lines show the trace of the main and splitter blades. Even if there is a shift in amplitude between the two maps, the flow structures are similar. A second example is given in Figure 7 which shows the pressure fluctuations over a rotor time period at three positions in the diffuser vane passage (Figure 3). The ensemble averaged measurements coming from the fast pressure sensors (dotted line) and the numerical results (continuous line) lead to signals which are very similar both in shape and strength. 
Therefore, the good agreement between numerical results and experiments allowed the use of the CFD results for investigating the effects of unsteadiness on the flow development within the diffuser.

4.1. Data Postprocessing. Nonuniformities and unsteadiness due to the rotor-stator interaction introduce major complexity in the analysis of the flow field. The problem can be simplified by decomposing the flow field, which beforehand requires the numerical data to be postprocessed.

The unsteady RANS simulation using the phase-lagged approach leads to the knowledge of the time-dependent data over a stator pitch. It is possible to reconstruct the field over a complete turn of the machine by using the spatial-temporal periodicity property of the flow field:

$$
f(r, z, \theta, t)=f\left(r, z, \theta+\frac{2 \pi}{N_{S}}, t+\frac{2 \pi}{\Omega_{R} N_{S}}\right) .
$$

The generated flow field which is unsteady but deterministic may be decomposed as proposed by Adamczyk et al. $[15,16]$. At given $(r, z)$ coordinates, this decomposition is written in the absolute frame of reference as follows:

$$
f(\theta, t)=\underbrace{f^{\mathrm{axi}}}_{(1)}+\underbrace{f^{S}(\theta)}_{(2)}+\underbrace{f^{R}\left(\theta-\Omega_{R} t\right)}_{(3)}+\underbrace{f^{\prime}(\theta, t)}_{(4)} .
$$

The first term is the axisymmetric field. Terms (2) and (3) represent the spatial fluctuations of the time-averaged field in the frame of reference of the vaned diffuser and impeller, respectively. Finally the term (4) represents the purely unsteady part of the field, which is time-dependent whatever the frame of reference.

An equivalent decomposition, based on Fourier transform, was proposed by Tyler and Sofrin [17]. The main result of this decomposition is the identification of the spatial harmonics $m$, which may be classified into three sets. The first set is composed of the $m=k N_{S}$ harmonics which represent the time-averaged flow in the absolute frame. The second set includes the $m=n N_{R}$ harmonics involved in the time-averaged flow field in the relative frame of reference. The third set includes the $m=n N_{R}+k N_{S}$ harmonics (with $n, k \neq 0)$ involved in the impeller diffuser interaction. The reconstructed signal from each set corresponds to the terms ( 2 ), (3) and (4) of (2), respectively. The angular rotation speed of the $m$ th spatial mode is given by

$$
\Omega_{m, n}=\frac{n N_{R}}{n N_{R}+k N_{S}} \Omega_{R}
$$

This decomposition was applied to the numerical data, coming from the unsteady computation, extracted at 50\% section height, at mid gap between the impeller trailing edge and the diffuser leading edge $\left(m^{*}=1.025\right.$, cf. Figure 2). It led to isolate the purely unsteady part (term (4) of (2)) involved in the interaction from the complete flow field. The rotation speeds of all the spinning modes contained in this unsteady part were shown to be organized into three main classes. The rotation speed of each class characterizes the type of transport of the associated structures. In the present case (at 50\% section height at mid-inter-row gap), the reduced values of the rotation speed of each class are given hereafter:

(i) rotation speed of convected structures:

$$
\Omega_{c}^{*}=\frac{\Omega_{c}}{\Omega_{R}} \approx \frac{V_{\theta}}{U} \Longrightarrow 0.53 \leq \Omega_{c}^{*} \leq 0.95,
$$

(ii) rotation speed of progressive pressure waves:

$$
\Omega_{p}^{+*}=\frac{\Omega_{p}^{+}}{\Omega_{R}} \approx \frac{V_{\theta}+c}{U} \Longrightarrow 1.05 \leq \Omega_{p}^{+*} \leq 2.12,
$$

(iii) rotation speed of regressive pressure waves:

$$
\Omega_{p}^{-*}=\frac{\Omega_{p}^{-}}{\Omega_{R}} \approx \frac{V_{\theta}-c}{U} \Longrightarrow-0.25 \leq \Omega_{p}^{-*} \leq 0.02 .
$$

The amplitude of the spinning modes of the unsteady pressure and entropy are plotted as a function of the reduced rotation speed in Figures 8(a) and 8(b). These variables were chosen amongst all because the unsteadiness mainly results from, on the one hand, the jet and wake flow structure emerging from the impeller, and on the other hand, the impeller-diffuser interaction including potential and shock wave interactions. The former source is well described using the entropy whereas the second source is highlighted using the pressure.

Regarding the pressure spectrum (Figure 8(a)), the dominant modes belong to the progressive and regressive modes classes which were expected because the pressure field is obviously dominated by pressure waves. Concerning the entropy spectrum (Figure 8(b)) the dominant modes belong to the convected class which was again expected because the entropy is a good tracer of the jet-wake structure. It has to be noted that the modes contained in each class are the modes which carry the physical structures whereas the modes which are outside the class act as modulators.

4.2. Physical Mechanisms Involved in the Interaction. The spinning modes included in each speed range are a representation, in the spectral plane, of aerodynamic structures moving, in the physical plane, by convection or propagation. Such a classification of the aerodynamic structures according to the type of transport proved to be efficient to identify the main mechanisms involved in the impeller-diffuser interaction [18]. Three main unsteady structures were identified: $\alpha^{+}, \alpha^{-}$pressure waves (propagated structures), and the classical jet/wake structure (convected structure). The entropy map (Figure 9) at impeller exit highlights the jet/wake structure characterized by low/high level of entropy, respectively. This structure is convected by the flow through the vaned diffuser. Due to the fact that the impeller rotates, this spatial distortion (in the impeller frame) becomes unsteady in the diffuser frame.

In order to highlight the fluctuations induced by the pressure waves, the pressure gradient in the flow direction was calculated: $(\vec{\nabla} p)_{s}=\vec{\nabla} p \cdot \vec{V} /\|\vec{V}\|$. The sign of that variable indicates if the pressure gradient has the same 


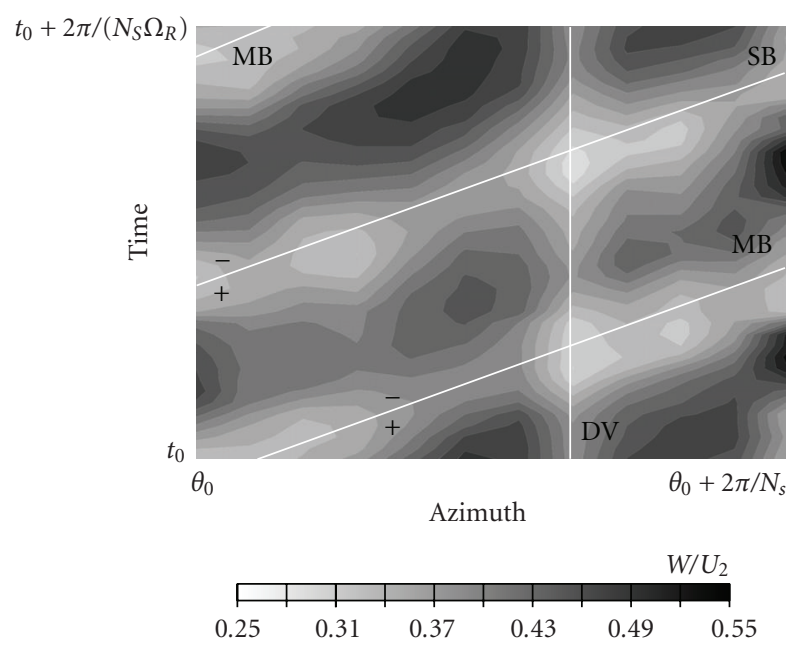

(a) LDA results

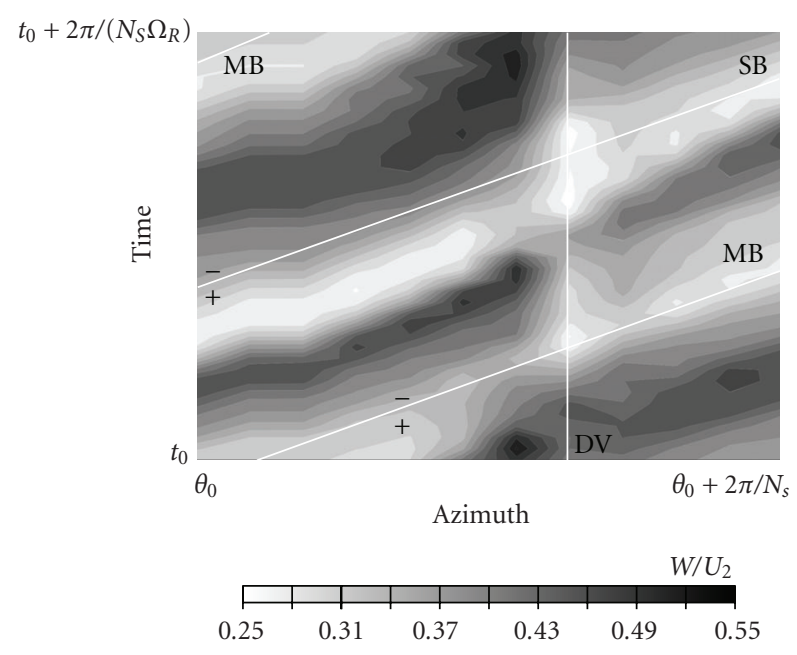

(b) numerical results

FigURE 6: Reduced relative velocity at mid inter row gap and 70\% section height, over a stator pitch and a rotor time period.

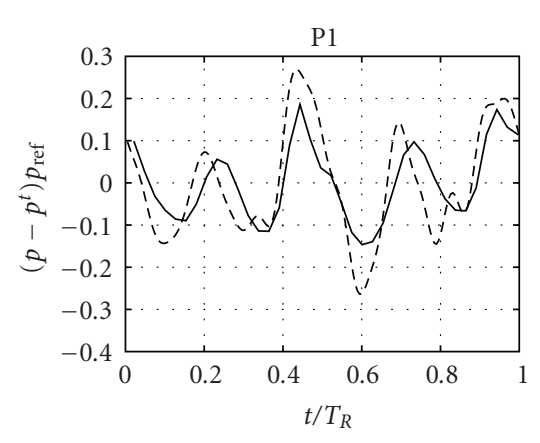

- Numerical data

- - Experimental data

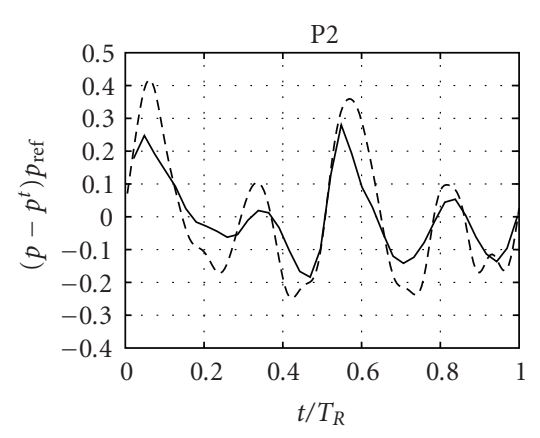

- Numerical data

- - - Experimental data

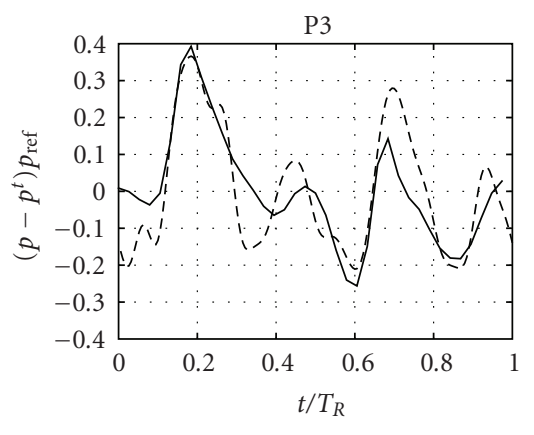

- Numerical data

- - Experimental data

FIGURE 7: Fluctuations of reduced pressure over a rotor time period at three sensor locations within the diffuser vane passage.

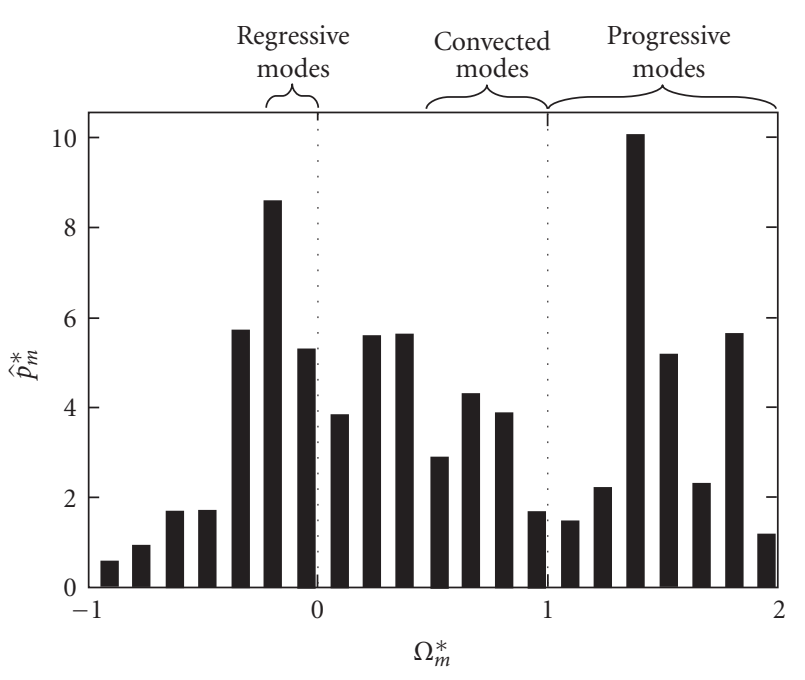

(a) unsteady pressure

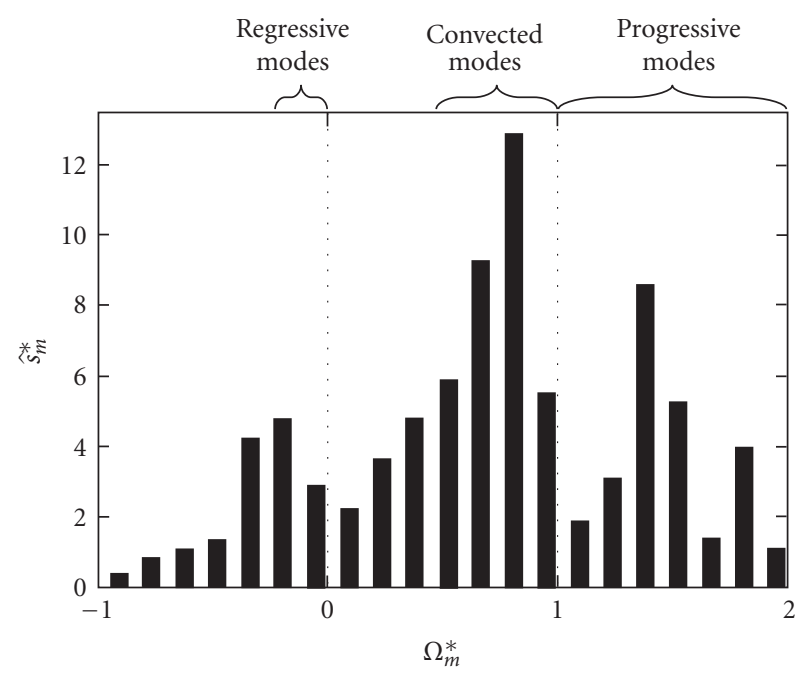

(b) entropy

Figure 8: Reduced amplitudes of the spinning modes at mid inter row gap and 50\% section height. 


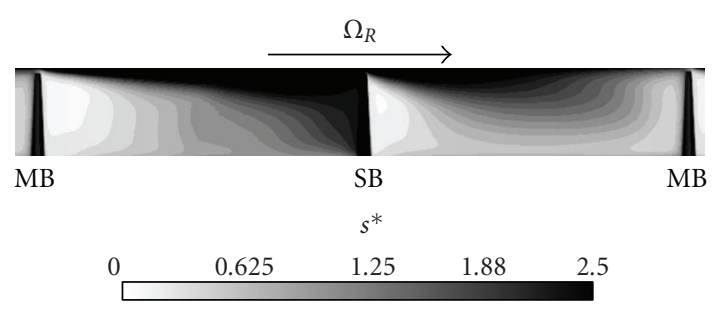

FIGURE 9: Reduced and time-averaged entropy at impeller exit.

direction as the velocity vector, which allows to identify the favorable or unfavorable pressure gradients. Figure 10 gives six time steps of the pressure gradient in the impeller-diffuser interaction zone, at $50 \%$ blade height. The white curve shows the vane leading edge bow shock wave. The black curves and doted white curves mark out the $\alpha^{+}$and $\alpha^{-}$waves, respectively. The first time step map (Figure 10(a)) shows the shock wave at a time just before its chopping by the impeller blade. At the next time step (Figure 10(b)), the trailing edge intersects the strong part of the shock wave which is thus reflected on the blade pressure surface, leading to an $\alpha^{+}$wave (noted $\alpha^{+}{ }_{2}$ ), then to an $\alpha^{-}$wave (visible in the third time step map, Figure 10(c)) emerging from reflection of the $\alpha^{+}$ wave on the vane leading edge. A wave noted $\alpha^{+}{ }_{1}$ may be observed in Figure 10(b). This wave propagates without any obstacle up to the fourth time step. At the fifth time step (Figure 10(e)), the wave passes the shock wave and hits the vane leading edge, leading to the $\alpha^{-}{ }_{1-b}$ wave which rotates backwards. In the last map (Figure 10(f)), the $\alpha^{+}{ }_{1}$ wave has been cut into two branches $\alpha^{+}{ }_{1-a}$ and $\alpha^{+}{ }_{1-b}$. The $\alpha^{+}{ }_{1-b}$ branch moves quicker than the $\alpha^{+}{ }_{1-a}$ branch because it is in a supersonic flow while $\alpha^{+}{ }_{1-a}$ is in a subsonic flow.

4.3. Development of the Unsteady Structures through the Diffuser. The jet and wake structure emerging from the impeller and the progressive pressure waves $\alpha^{+}$brought about by the interaction between the vane bow shock wave and the impeller blade move downstream through the diffuser. Their effects on the flow structure that develops within the diffuser are hereafter discussed.

4.3.1. $2 D$ Analysis. Figure 11 gives at a given time step the color map of the unsteady entropy reduced by its time-averaged value in the vaned diffuser at midsection height. The velocity fluctuations are superimposed with black arrows. The jet/wake material corresponds to the low/high entropy value (resp., blue/red color). The spatial correlation between the velocity fluctuations and the jet and wake indicates that the wake is transported from suction side to pressure side whereas the jet moves from pressure side to suction side. The result is an accumulation of low momentum fluid toward the vane pressure side.

The color map of the unsteady pressure reduced by its time-averaged value (Figure 12) shows the influence of the $\alpha^{+}$waves on the velocity fluctuations which are plotted with black arrows. The black curves mark out the locations of the crests of the $\alpha^{+}$waves. The pressure gradient of an $\alpha^{+}$upward wavefront leads to positive velocity fluctuations which order of magnitude may reach $25 \%$ of the mean velocity; it is therefore a significant unsteady energy source. The opposite effect is obviously achieved by a forward wavefront.

It may be observed in Figure 12 that the $\alpha^{+}$waves are stronger near the suction side than near the pressure side which is inherent to their development process. Actually the $\alpha^{+}$wave development may be decomposed into three parts as shown in Figure 13. In the first part, the $\alpha^{+}$wave is generated by the reflection of the shock wave on the impeller blade pressure side. It is all the more strong that located close to the vane leading edge, that is, where the bow shock wave is the strongest. As the $\alpha^{+}$wave moves downstream (part 2) its propagation is spatially blocked by the vane suction side whereas it propagates freely on the other side. Within the vane passage (part 3) the concave curvature of the suction side reinforces the strength of the wave whereas the convex curvature of the pressure side reduces it.

Figure 14 gives the pressure gradient in the flow direction. The black color corresponds to a $\alpha^{+}$upward wavefront and the white to a downward wavefront. The dotted curves mark the locations of the wave crests. Finally the continuous black curves locate the zero isovalue of the azimuthal velocity which delineates a separated boundary layer. Despite the weakness of the $\alpha^{+}$waves near the vane pressure side, their downward wavefronts generate a separation of the boundary layer which is unable to resist to the adverse pressure gradient. Thus, the boundary layer which develops on the convex pressure side separates at the downward wavefront passage and reattaches at the upward wavefront passage, leading to a pulsating behavior. As moving downstream, the size of the unsteady separation bubbles increases due to the decrease in mean velocity.

4.3.2. 3D Analysis. The hub-to-shroud flow evolution is first analyzed by calculating the energy of the spectra of the spinning modes according to their type of transport (convection, progressive propagation, and regressive propagation). The energy of the three spinning mode classes as a function of the reduced blade height is calculated as follows:

(i) energy of convected structures:

$$
\begin{gathered}
\xi_{c_{(f)}}\left(h^{*}\right)=\sum_{\Omega_{m, n}}\left(\hat{f}^{2}\left(\Omega_{m, n}, h^{*}\right)\right) \\
\text { with } \min \left(\frac{V_{\theta}}{U}\left(h^{*}\right)\right)<\frac{\Omega_{m, n}}{\Omega_{R}}<\max \left(\frac{V_{\theta}}{U}\left(h^{*}\right)\right),
\end{gathered}
$$

(ii) energy of progressive pressure waves:

$$
\begin{gathered}
\xi_{p_{(f)}^{+}}\left(h^{*}\right)=\sum_{\Omega_{m, n}}\left(\hat{f}^{2}\left(\Omega_{m, n}, h^{*}\right)\right) \\
\text { with } \min \left(\frac{V_{\theta}+c}{U}\left(h^{*}\right)\right)<\frac{\Omega_{m, n}}{\Omega_{R}}<\max \left(\frac{V_{\theta}+c}{U}\left(h^{*}\right)\right),
\end{gathered}
$$




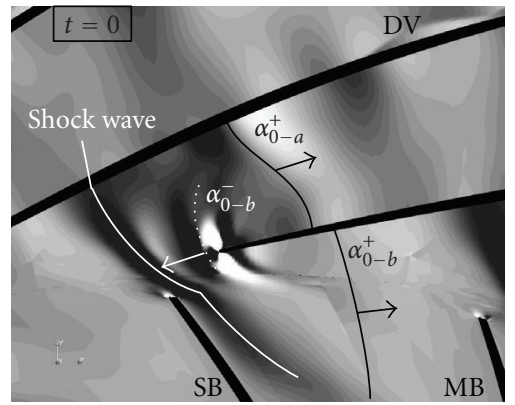

(a)

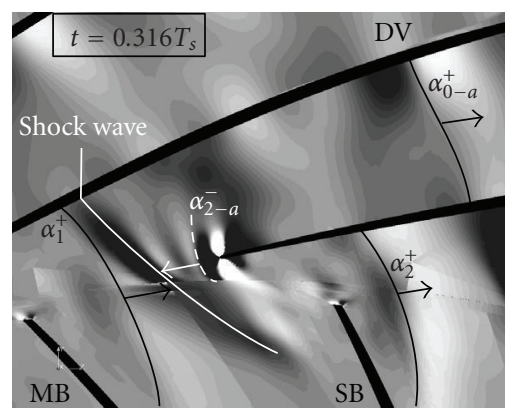

(d)

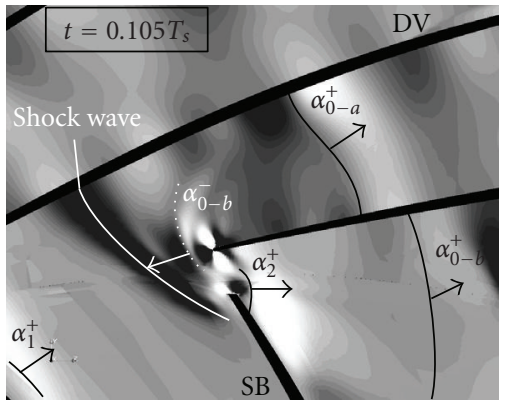

(b)

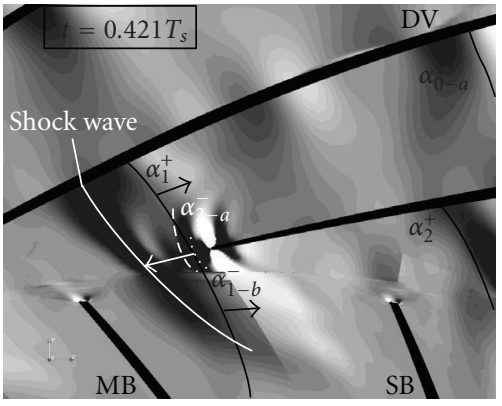

(e)

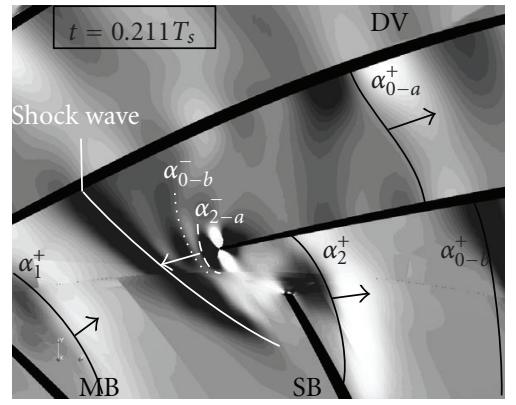

(c)

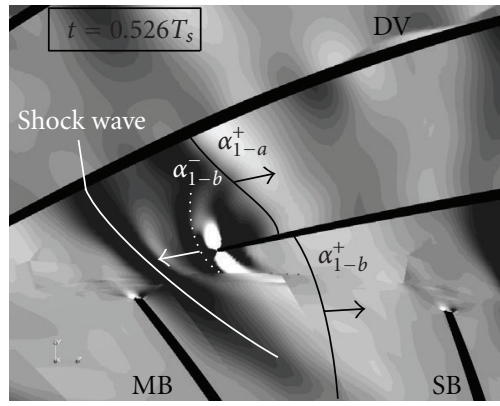

(f)

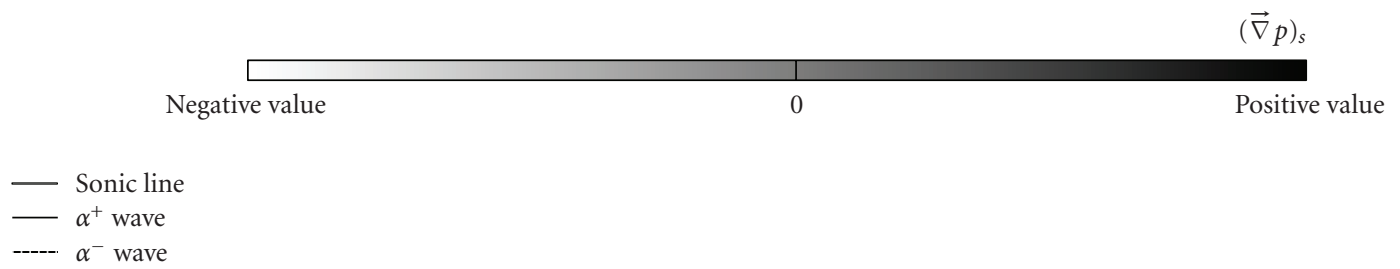

FIGURE 10: Generation of pressure waves at six time steps.

(iii) energy of regressive pressure waves:

$$
\xi_{p_{(\mathrm{f})}^{-}}\left(h^{*}\right)=\sum_{\Omega_{m, n}}\left(\hat{f}^{2}\left(\Omega_{m, n}, h^{*}\right)\right)
$$

with $\min \left(\frac{V_{\theta}-c}{U}\left(h^{*}\right)\right)<\frac{\Omega_{m, n}}{\Omega_{R}}<\max \left(\frac{V_{\theta}-c}{U}\left(h^{*}\right)\right)$.

Figure 15 gives the evolution of the energy contained in each class (reduced by the total energy at 50\% section height) versus the reduced section height $\left(h^{*}=0\right.$ at hub, $h^{*}=1$ at shroud) at mid inter row gap. The considered spectra are the unsteady pressure field (Figure 15(a)) and entropy (Figure 15(b)). The dotted, fine dotted, and full lines represent the regressive, convected, and progressive classes, respectively.

Due to the intrinsic nature of the pressure and entropy, the dominant class in the pressure field corresponds to the modes which are propagated in a progressive way, whereas it corresponds to the modes which are convected in the entropy field. The hub-to-shroud evolution shows that the progressive propagated structures have no significant variation compared to the convected structures (especially regarding the entropy, Figure 15(b)). That reveals a homogeneous strength of the $\alpha^{+}$waves all over the span which are thus two-dimensional waves. The hub-to-shroud evolution of the energy of the entropy spectrum shows two maxima. One is located at around $h^{*}=0.95$; it is due to the tip leakage flow. The other one which spreads from around 10 to $70 \%$ of section height is due to the jet/wake structure.

Figure 16 gives at a given time step the color map of the reduced unsteady entropy at midsection height within the diffuser passage. The black and white contours indicate the sign of the hub-to-shroud velocity component: inside the black contours the flow is transported toward the shroud whereas inside the white contours the flow moves toward the hub. A strong correlation between the hub-toshroud migration zones and the low/high entropy area is clearly observed. Actually the jet migrates toward the shroud whereas the wake migrates toward the hub.

Merging this conclusion with the fact that the wake moves toward the pressure side (cf. Section 4.3.1), it is shown that there is an accumulation of low momentum fluid in the hub/pressure side corner. 


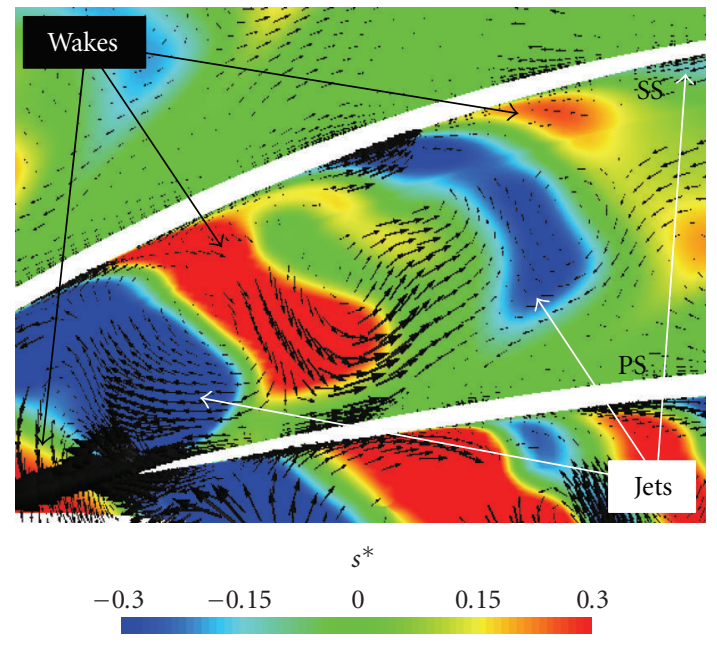

FIGURE 11: Color map of the reduced unsteady entropy and velocity fluctuations with arrows at a given time step, at midsection height.

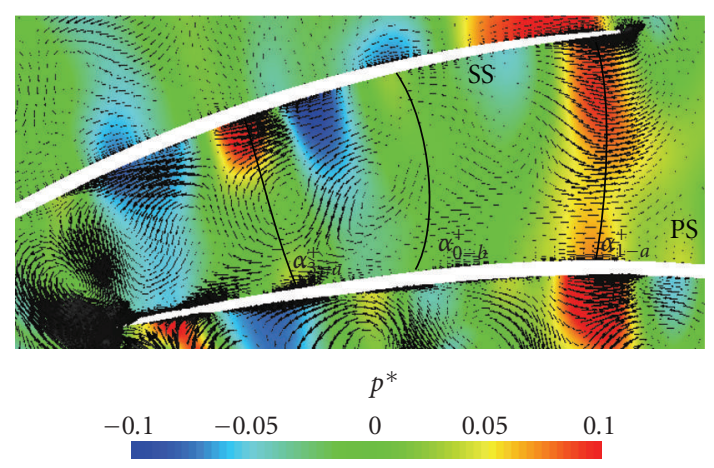

FIGURE 12: Color map of the reduced unsteady pressure and velocity fluctuations with arrows at a given time step, at midsection height.

Figure 17 shows the pressure gradient in the flow direction at an arbitrary time step. Figure $17(\mathrm{a})$ is extracted at a reduced blade height $h^{*}=0.02$ (just over the hub boundary layer). Figure 17(c) is extracted at $h^{*}=0.98$ (just under the shroud boundary layer). The mid-height pattern is given in Figure $17(\mathrm{~b})$ as reference. The dotted and fine dotted curves symbolize the $\alpha^{+}$waves location (branches $a$ and $b$, resp.). The shape and strength of the $\alpha^{+}$waves are similar whatever the blade height, which reveals again a $2 \mathrm{D}$ structure of these pressure waves. Therefore, the change in the flow field from hub-to-shroud results from the change in momentum from hub-to-shroud, as highlighted in Figure 15. The black contours in Figure 17 mark the zero isovalue of the azimuthal velocity, which gives a qualitative separation criterion. Three kinds of boundary layer separation may be distinguished depending on the mechanisms which trigger them .

Separation of Kind 1. The separation is due to an overincidence of the space and time-averaged flow at the diffuser inlet. Figure 18 gives the hub-to-shroud evolution of the axisymetric incidence: above $75 \%$ section height, the over-incidence triggers a vane pressure side boundary

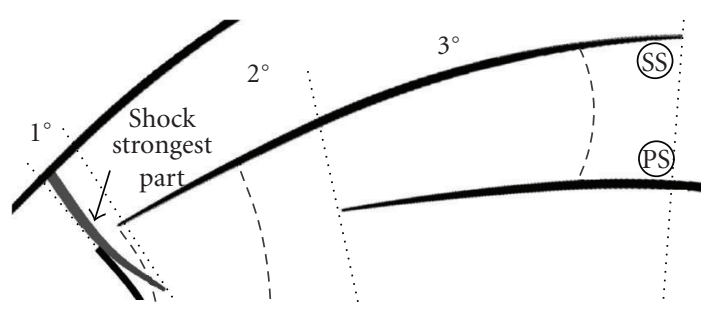

FIGURE 13: Sketch of the $\alpha^{+}$wave development.
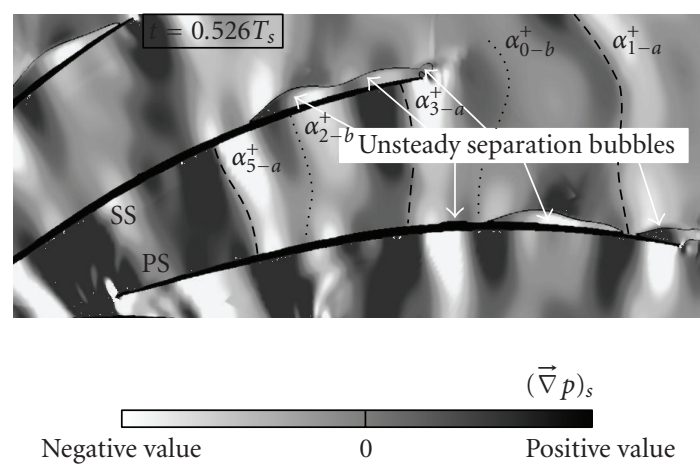

FIgURe 14: Pressure gradient in the flow direction at 50\% height. Black contour: zero-isovalue of the azimuthal velocity $\mathrm{V}_{\theta}$.

layer separation which exists whatever the time. It is thus considered as a steady separation.

Separation of Kind 2. The shock wave-boundary layer interaction on the vane suction side leads to a separation of the boundary layer in the hub and shroud corners (Figures 17(a) and $17(\mathrm{c}))$. This separation permanently exists; it is thus again considered as steady.

Separation of Kind 3. The vane diffuser pressure side convex curvature weakens the boundary layer. The successive passing of the $\alpha^{+}$waves leads to a pulsating separation of the vane pressure side boundary layer at mid hub-to-shroud distance (Figure 17(b)).

At $h^{*}=2 \%$ (Figure 17(a)), the vane pressure surface boundary layer is always separated along $85 \%$ of the vane chord. It results from the migration of the wake material which accumulates low momentum fluid in the vane pressure side-hub corner. The passing of the $\alpha^{+}$waves only varies the intensity of the separation.

At 98\% height (Figure 17(c)) a separation is observed on the rear part of the vane due to the low momentum material in the pressure side-shroud corner. Nevertheless no separation exists at around mid-chord because of the migration of the jet toward the shroud which energizes the pressure side boundary layer.

Finally, it may be concluded that the separation located near the hub and shroud reveals a steady state (kind 3steady), whereas at midsection height the separation is fully unsteady (kind 3-unsteady). 


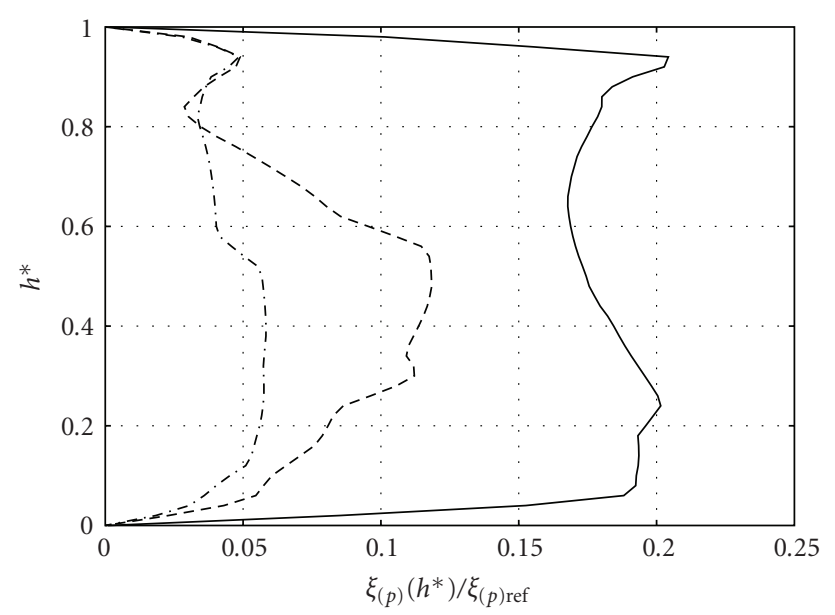

(a) unsteady pressure

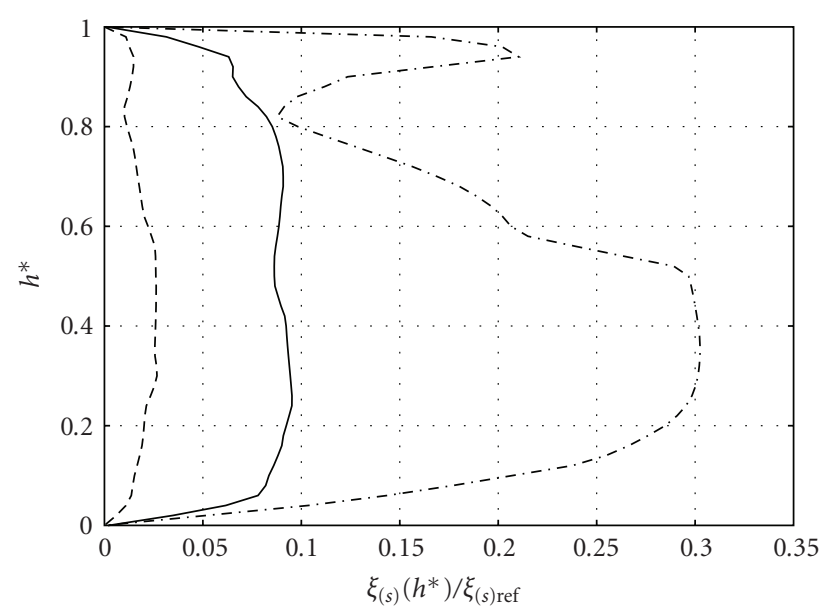

(b) unsteady entropy

FIGURE 15: Hub-to-shroud evolution of the spinning modes energy according to their type of transport, at mid inter row gap.

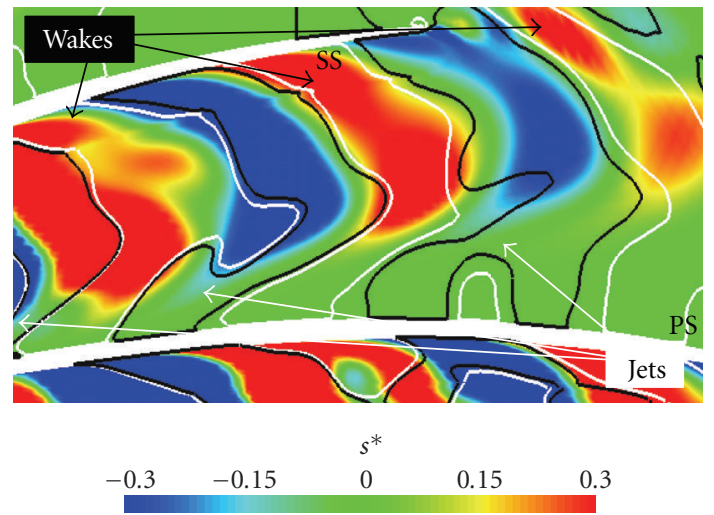

FIGURE 16: Color map of the reduced unsteady entropy, black/white contours: migration toward shroud/hub at a given time step, at midsection height.

\section{Conclusion}

The unsteady flow that develops in the vaned diffuser of a transonic high-pressure-ratio centrifugal compressor has been analyzed in detail from CFD results. The unsteadiness comes from the combined effects of the jet/wake structure (emerging from the impeller flow) and the $\alpha^{+}$pressure waves brought about by the interaction between the vane bow shock wave and the impeller blade.

Within the diffuser, whereas the pressure waves are similar in shape and strength whatever the blade height, the jet and wake migrate toward shroud and hub, respectively. Therefore, the nonuniformity of the flow field, along the blade height, is essentially attributable to the low/high momentum fluid convection. Various separated zones were highlighted which state depends on the mechanisms which trigger the separation. The boundary layer separation on the vane pressure side revealed a steady state near hub and shroud, whereas it had a purely unsteady behavior at mid span.

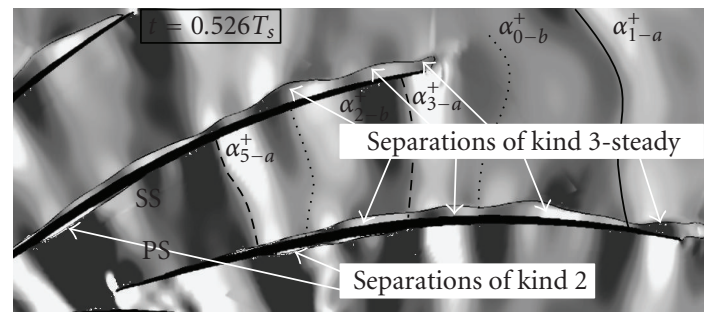

(a)

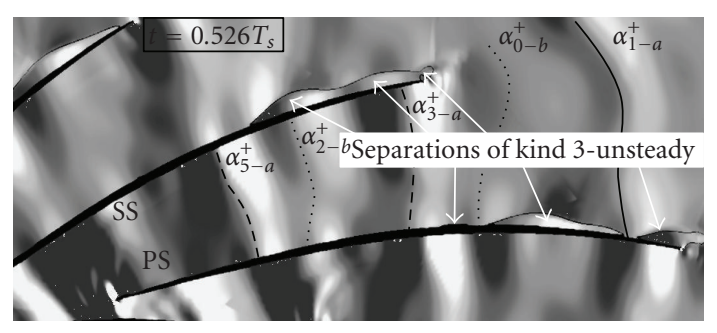

(b)

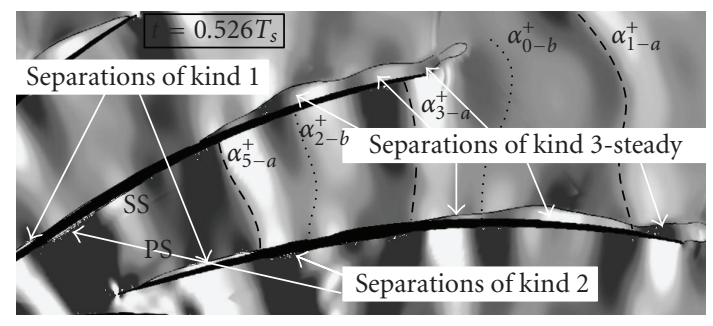

$(\vec{\nabla} p)_{s}$

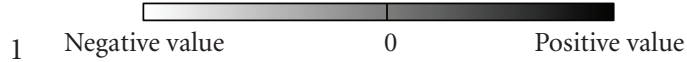

(c)

Figure 17: Pressure gradient in the flow direction at 2\% (a), 50\% (b), and 98\% (c) section height black contour: zero-isovalue of the azimuthal velocity $V_{\theta}$. 


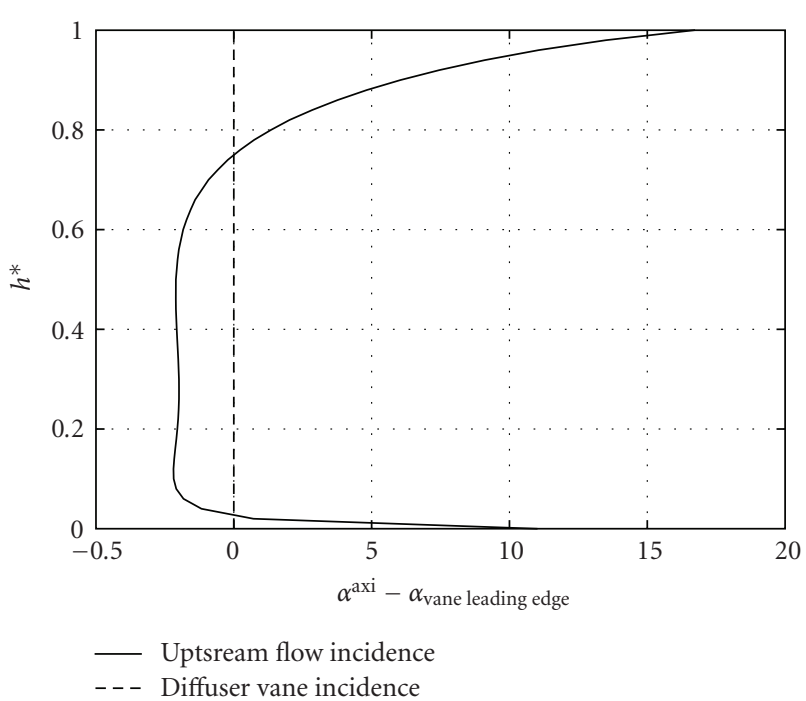

FIGURE 18: Evolution of the axisymetric Incidence at diffuser inlet from hub-to-shroud.

Finally, even if the diffuser performance is mainly governed by the incidence of the axisymetric flow as stated in particular by Shum et al. [6], the present results show the importance of the unsteady structures on the internal diffuser flow.

\section{Nomenclature}

$\begin{array}{ll}c: & \text { sound celerity } \\ f: & \text { arbitrary flow variable } \\ h: & \text { blade height } \\ k, l: & \text { kinetic turbulent energy, scale of } \\ & \text { turbulence size } \\ L D A: & \text { Laser Doppler Anemometry } \\ M B, S B: & \text { main blade, splitter blade } \\ m, n: & \text { meridional coordinate or spinning } \\ N: & \text { mode } \\ P S, S S: & \text { pressure side, suction side } \\ p: & \text { pressure } \\ r, \theta, z: & \text { cylindrical coordinates } \\ s: & \text { entropy } \\ T: & \text { time period } \\ t: & \text { time } \\ U: & \text { circumferential impeller velocity } \\ V, W: & \text { absolute, relative velocity }\end{array}$

\section{Greek Letters}

$\alpha$ : pressure waves

$\xi$ : energy

$\pi$ : pressure ratio

$\Omega$ : rotation speed

\section{Superscripts}

\author{
+/-: progressive/regressive pressure \\ waves \\ *: $\quad$ reduced variable \\ axi: axisymetric variable
}

\section{Subscripts}

$\begin{array}{ll}0: & \begin{array}{l}\text { stagnation variable } \\ \text { convected structures }\end{array} \\ c: & \begin{array}{l}\text { meridional, radial, azimuthal, } \\ \text { curvilinear component }\end{array} \\ m, r, \theta, & \text { mode } \\ \text { nominal } & \text { nominated structures } \\ \mathrm{P}: & \text { propagated } \\ R / S: & \text { rotor/stator (impeller/diffuser) }\end{array}$

\section{Acknowledgments}

The authors would like to thank TURBOMECA which supported this research, together with ONERA which collaborated on the numerical simulation. Dr. Pascale Kulisa (LMFA) is acknowledged for numerical support. Gilbert Halter, Patrick Krikorian, Benoît Paoletti and Sébastien Goguey (LMFA) are acknowledged for technical support.

\section{References}

[1] H. Krain, "A study on centrifugal impeller and diffuser flow," Journal of Engineering for Power, vol. 103, no. 4, pp. 688-697, 1981.

[2] M. Inoue and N. A. Cumpsty, "Experimental study of centrifugal impeller discharge flow in vaneless and vaned diffusers," Journal of Engineering for Gas Turbines and Power, vol. 106, no. 2, pp. 455-467, 1984.

[3] K. U. Ziegler, H. E. Gallus, and R. Niehuis, "A study on impeller-diffuser interaction-part I: influence on the performance," Journal of Turbomachinery, vol. 125, no. 1, pp. 173-182, 2003.

[4] K. U. Ziegler, H. E. Gallus, and R. Niehuis, "A study on impeller-diffuser interaction-part II: detailed flow analysis," Journal of Turbomachinery, vol. 125, no. 1, pp. 183-192, 2003.

[5] S. Deniz, E. M. Greitzer, and N. A. Cumpsty, "Effects of inlet flow field conditions on the performance of centrifugal compressor diffusers_-part 2: straight-channel diffuser," Journal of Turbomachinery, vol. 122, no. 1, pp. 11-21, 2000.

[6] Y. K. P. Shum, C. S. Tan, and N. A. Cumpsty, "Impellerdiffuser interaction in a centrifugal compressor," Journal of Turbomachinery, vol. 122, no. 4, pp. 777-786, 2000.

[7] H. Krain, "Unsteady diffuser flow in a transonic centrifugal compressor," International Journal of Rotating Machinery, vol. 8, no. 3, pp. 223-231, 2002.

[8] H. Krain and C. Hah, "Numerical and experimental investigation of the unsteady flow field in a transonic centrifugal compressor," in Proceedings of the International Gas Turbine Congress (IGTC '03), Tokyo, Japan, November 2003, IGTC2003Tokyo TS-049.

[9] M. P. Wernet, M. M. Bright, and G. J. Skoch, "An investigation of surge in a high-speed centrifugal compressor using digital PIV," Journal of Turbomachinery, vol. 123, no. 2, pp. 418-428, 2001. 
[10] Z. S. Spakovszky, "Backward traveling rotating stall waves in centrifugal compressors," Journal of Turbomachinery, vol. 126, no. 1, pp. 1-12, 2004.

[11] D. Bonaiuti, A. Arnone, C. Hah, and H. Hayami, "Development of secondary flow field in a low solidity diffuser in a transonic centrifugal compressor stage," in Proceedings of the ASME Turbo Expo, pp. 445-454, Amsterdam, The Netherlands, June 2002.

[12] N. Rochuon, Analyse de l'écoulement tridimensionnel instationnaire dans un compresseur centrifuge à fort taux de pression, Ph.D. thesis, Ecole Centrale de Lyon, Cedex, France, 2007.

[13] L. Cambier and M. Gazaix, "elsA: an efficient object-oriented solution to CFD complexity," in Proceedings of the 40th AIAA Aerospace Science Meeting and Exhibit, Reno, Nev, USA, January 2002.

[14] B. R. Smith, "Prediction of hypersonic shock wave turbulent boundary layer interactions with the $k-l$ two equaton turbulence model," in Proceedings of the 33th AIAA, Aerospace Sciences Meeting and Exhibition, Reno, Nev, USA, January 1995.

[15] J. J. Adamczyk, "Model equation for simulating flows in multistage turbomachinery," Technical Memorandum 86869, NASA, Cleveland, Ohio, USA, November 1984.

[16] J. J. Adamczyk, R. A. Mulac, and M. L. Celestina, "A model for closing the inviscid form of the average-passage equation system," ASME paper 86-GT-227, 1986.

[17] J. M. Tyler and T. G. Sofrin, "Axial flow compressor noise studies," SAE Transactions, vol. 70, pp. 309-332, 1962.

[18] S. Callot, P. Ferrand, and S. Aubert, "An analysis of the spatiotemporal periodicity based on a full stage computation of a transonic turbine," in Proceedings of the 9th International Symposium on Aerodynamics, Aeroacoustics and Aeroelasticity of Turbomachines (ISUAAAT 'O0), Lyon, France, September 2000. 

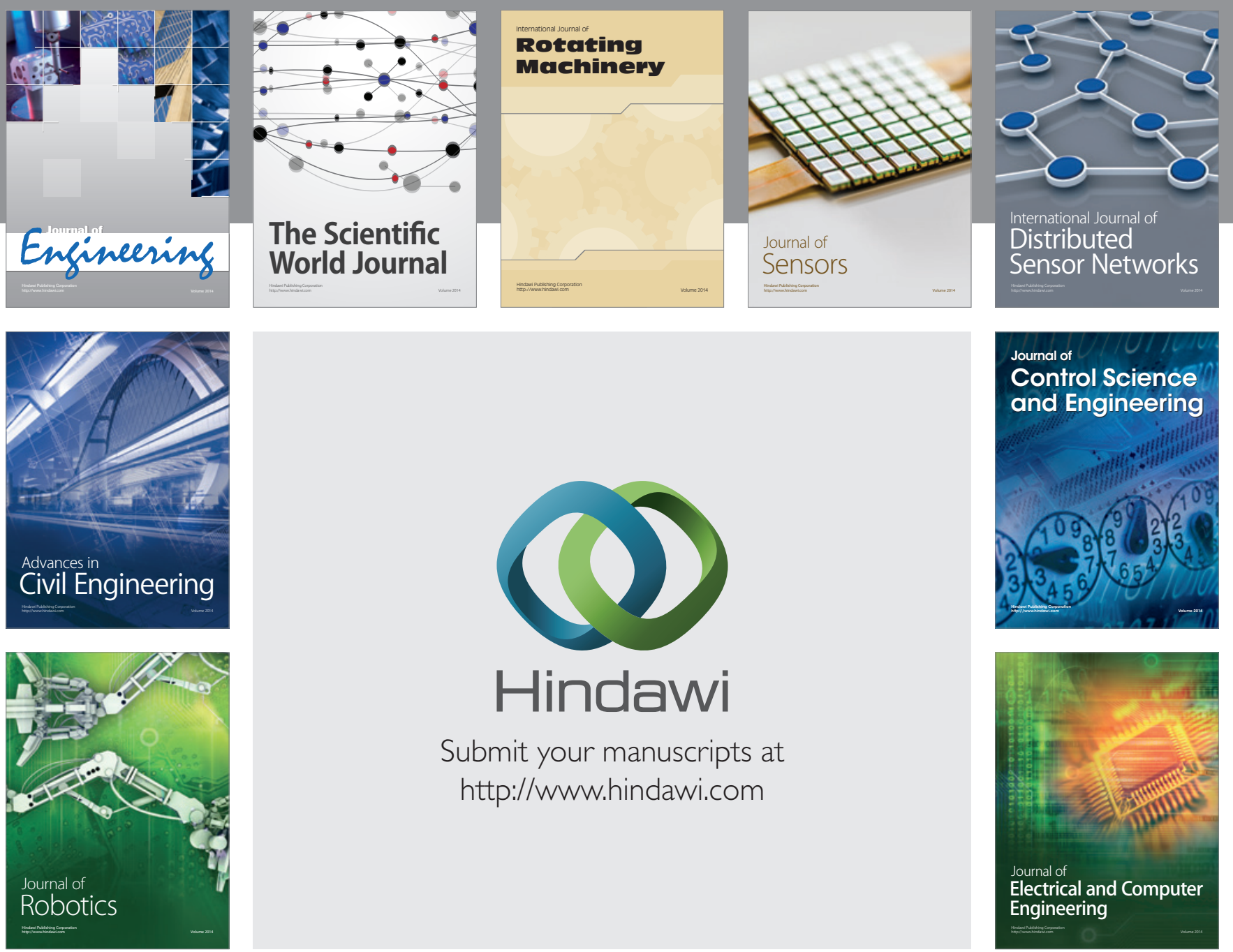

Submit your manuscripts at

http://www.hindawi.com
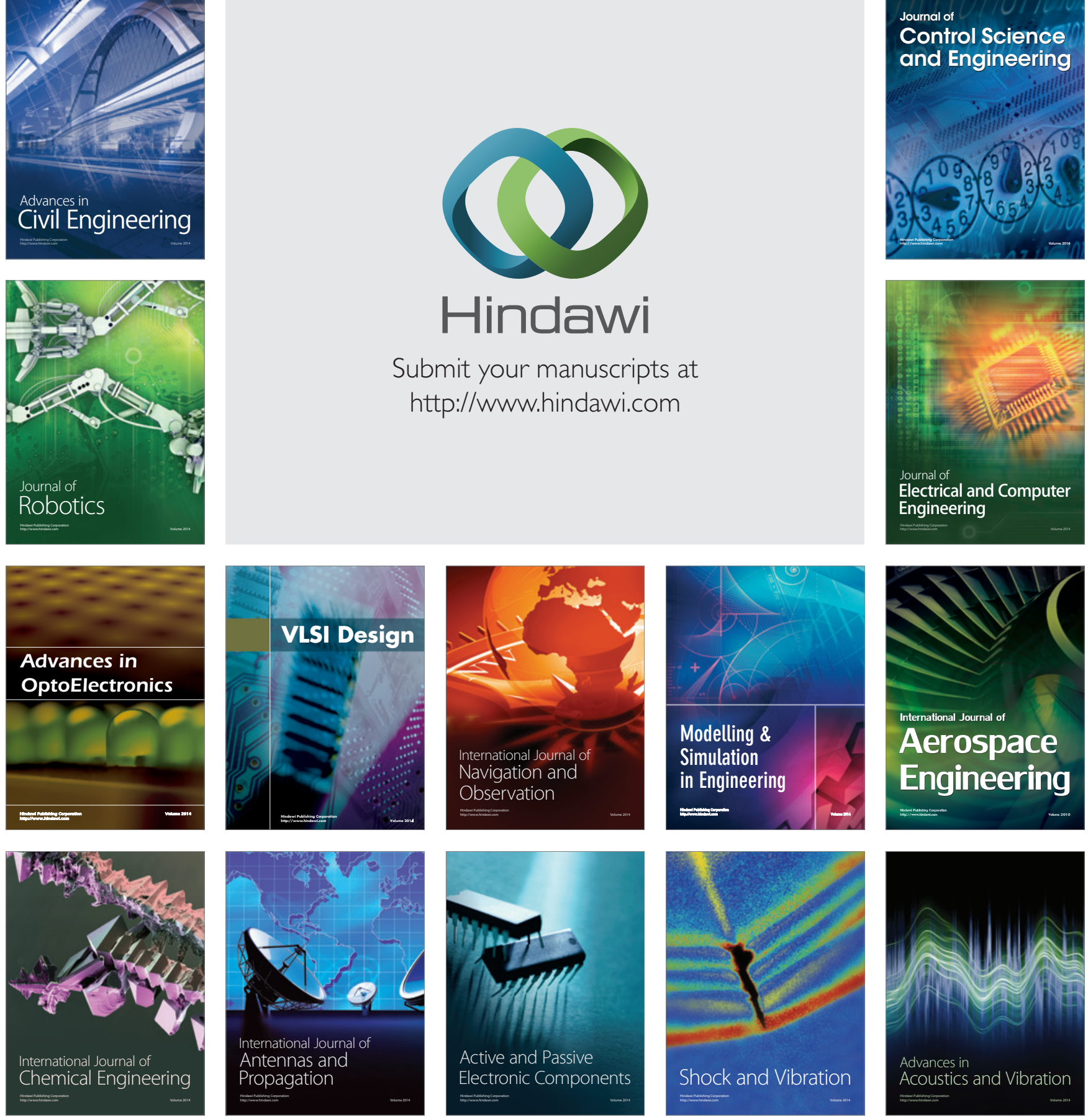